\title{
THE GREEN FUNCTION OF TEICHMÜLLER SPACES WITH APPLICATIONS
}

\author{
SAMUEL L. KRUSHKAL
}

\begin{abstract}
We describe briefly a new approach to some problems related to Teichmüller spaces, invariant metrics, and extremal quasiconformal maps. This approach is based on the properties of plurisubharmonic functions, especially of the plurisubharmonic Green function.

The main theorem gives an explicit representation of the Green function for Teichmüller spaces by the Kobayashi-Teichmüller metric of these spaces. This leads to various applications. In particular, this gives a new characterization of extremal quasiconformal maps.
\end{abstract}

I want to describe here briefly a somewhat new approach to certain subjects related to the Teichmüller theory, hyperbolic geometry, and quasiconformal maps. This approach is based on the properties of plurisubharmonic functions, especially of the Green function.

\section{The GreEN FUnCtion}

Recall that the Green function $g_{D}(x, y)$ of a domain $D$ in a complex Banach space $\mathscr{X}$ is defined as

$$
g_{D}(x, y)=\sup u_{y}(x) \quad(x, y \in D),
$$

where supremum is taken over all plurisubharmonic functions $u_{y}: D \rightarrow[-\infty, 0)$ having the representation $u_{y}(x)=\log \|x-y\|+O(1)$ in a neighborhood of the point $y$; here $\|\cdot\|$ is the norm on $\mathscr{X}$, and $O(1)$ is bounded above. This definition is easily extended to complex Banach manifolds.

The important properties of the Green function are:

(i) $g_{D}(x, y)$ does not increase under holomorphic maps from $D$;

(ii) $g_{D}(x, y)$ is plurisubharmonic in $x$ on $D$ for every fixed $y \in D$;

(iii) for the domains $D \subset \mathbf{C}^{n}$, the function $g_{D}(x, y)$ (provided it belongs to $L^{\infty}, \operatorname{loc}(D \backslash\{y\})$ is maximal and is a generalized solution of the homogeneous Monge-Ampére equation $\left(d d^{c} u\right)^{n}=0$ in $D \backslash\{y\}$.

(For details and other properties we refer to $[2,7,11,14]$.)

\section{MAIN THEOREM}

Now let us consider the Teichmüller space $T(\Gamma)$ of an arbitrary Fuchsian group $\Gamma$, for simplicity, without torsion. It is well known that $T(\Gamma)$ admits a structure of a complex (Banach) manifold; moreover, it can be holomorphically embedded as a bounded domain into some complex Banach space (see, e.g., [5, 12]).

\section{Our main result states:}

Received by the editors November 1, 1991.

1991 Mathematics Subject Classification. Primary 30C75, 31C10, 32G15, 32H15; Secondary 32F05, 32F15. 
Theorem 1. The Green function $g_{T(\Gamma)}(x, y)$ of the space $T(\Gamma)$ is given by

$$
g_{T(\Gamma)}(x, y)=\log \frac{e^{2 d_{T(\Gamma)}(x, y)}-1}{e^{2 d_{T(\Gamma)}(x, y)}+1}
$$

where $d_{T(\Gamma)}$ is the Kobyashi-Teichmüller metric on $T(\Gamma)$.

\section{SOME COROLLARIES}

Taking into account the known properties of the Kobayashi-Teichmüller metric, one derives from (2) the following remarkable consequences.

Corollary 1. The function $g_{T(\Gamma)}$ is symmetric with respect to its arguments

$$
g_{T(\Gamma)}(x, y)=g_{T(\Gamma)}(y, x) .
$$

Corollary 2. For the finite-dimensional Teichmüller space $T(p, n)$ (which corresponds to the Riemann surfaces of finite conformal type $(p, n))$, the Green function $g_{T(\Gamma)}(x, y)$ is $C^{1}$-differentiable on $T(p, n) \times T(p, n) \backslash\{$ diagonal $\}$.

The latter result is of special interest because the known general results about the Green function $g_{D}$ state at most the continuity of $g_{D}$ on $D \times D$, even in the case of domains $D$ in $\mathbf{C}^{n}$ with $C^{\infty}$-boundaries.

Corollary 2 follows from equality (2) and the $C^{1}$-differentiability of the Teichmüller metric for the spaces $T(p, n)$ established in [3,5]. On the other hand, it is well known (see $[5,16])$ that $d_{T(\Gamma)}$ is not $C^{2}$-differentiable (along some directions in $T(p, n))$; hence, $g_{T(\Gamma)}$ also is not $C^{2}$-differentiable in $x$ on $T(p, n) \backslash\{y\}$.

Now let us consider an extremal Beltrami differential $\mu(z) d \bar{z} / d z$ on the Riemann surface $X$ represented by the point $x$ in $T(\Gamma)$ that realizes the distance $d_{T(\Gamma)}(x, y)$; that is, having denoted $k(x, y)=\|\mu\|_{L^{\infty}}$ we get

$$
d_{T(\Gamma)}(x, y)=\frac{1}{2} \log \frac{1+k(x, y)}{1-k(x, y)} .
$$

Then equality (2) assumes the form

$$
g_{T(\Gamma)}(x, y)=\log k(x, y) .
$$

Corollary 3. The function $k(x, y)$, for every fixed $y$, is logarithmically plurisubharmonic in $x$ on $T(\Gamma)$.

Corollary 4. Every Teichmüller space $T(\Gamma)$ is complex hyperconvex (that means there exists a negative plurisubharmonic function $u(x)$ on $T(\Gamma)$ which tends to zero when $x$ tends to infinity).

The question of the complex hyperconvexity of Teichmüller spaces was stated by $M$. Gromov and is solved in the affirmative for the finite-dimensional Teichmüller spaces $T(p, n)$ in [9].

Let us add here that the function $\log \kappa(x)$ constructed in [9] for the spaces $T(p, n)$ using the Grunsky coefficient inequality is related to the Green function $g_{T(\Gamma)}(x, 0)$ with strong inequality $\log \kappa(x)<g_{T(\Gamma)}(x, 0)$ and seems to 
be a minimal negative plurisubharmonic function on $T(p, n)$ with prescribed singularity at the origin.

\section{A SKETCH OF THE PROOF OF THEOREM 1}

Before deriving other corollaries of Theorem 1, we shall give a sketch of its proof. For this we must use an alternative definition of the Green function proposed by Poletskii for finite-dimensional domains (see [14]).

Let $D$ be a domain in a complex Banach space $\mathscr{X}$. For the given points $x, y \in D$, consider the holomorphic maps $f$ from the unit disc $\Delta=\{t \in \mathbb{C}$ : $|t|<1\}$ into $D$ with $f(0)=x$ and denote

$$
v_{f}(x, y)=\sum_{\zeta_{j} \in f^{-1}(y)} k_{j} \log \left|\zeta_{j}\right|,
$$

where the sum (which may be equal to $-\infty$ ) is taken over all preimages $\zeta_{j} \in \Delta$ of the point $y$, and $k_{j}$ is the multiplicity of $f$ at $\zeta_{j}$. Now put

$$
\tilde{g}_{D}(x, y)=\inf \left\{v_{f}(x, y): f \in \operatorname{Hol}(\Delta, D), f(0)=x\right\} \text {. }
$$

Poletskii proved (see [14]) that for the domains in $\mathbf{C}^{n}$ the function $\tilde{g}_{D}$ coincides with the Green function $g_{D}$ defined above by (1). His proof uses essentially some properties of holomorphic functions known for the finitedimensional case only. Thus we must use a different approach.

We start by proving the following

Lemma 1. Let $B=B\left(x_{0}, r\right)$ denote the ball $\left\{x \in \mathscr{X}:\left\|x-x_{0}\right\|<r\right\}$. For all $(x, y) \in B \times B$ the equality $\tilde{g}_{B}(x, y)=g_{B}(x, y)$ holds.

Assume now without loss of generality that $\Gamma$ acts discontinuously on the upper (and lower) half plane $U=\{z: \operatorname{Im} z>0\}$. We shall apply Lemma 1 to the unit ball $M(\Gamma)$ of the space of Beltrami differentials with respect to $\Gamma$, supported in $U$, with $L^{\infty}$-norm.

The next considerations are different for the cases when $\operatorname{dim} T(\Gamma)<\infty$ and $\operatorname{dim} T(\Gamma)=\infty$, respectively.

In the case $\operatorname{dim} T(\Gamma)<\infty$ we can use the indicated result of Poletskii. It is sufficient to prove (2) for $y=0$, the general case following from this if one uses a standard right translation from $T(\Gamma)$ to some $T\left(\Gamma^{\prime}\right)$. Applying Lemma 1 and Slodkowski's theorem on the extension of holomorphic motions [17], we derive

Lemma 2. For all $x \in T(\Gamma)$ the equality

$$
g_{T(\Gamma)}(x, 0)=\inf \left\{g_{M(\Gamma)}(\mu, 0): \Phi_{\Gamma}(\mu)=x\right\},
$$

holds where $\Phi_{\Gamma}$ is the canonical projection $M(\Gamma) \rightarrow T(\Gamma)$.

Now, using Royden's theorem about the coincidence of Kobayashi and Teichmüller metrics on $T(\Gamma)$ (see [16],[4]) and some properties of the Green function, we obtain (2).

In the case $\operatorname{dim} T(\Gamma)=\infty$ one must approximate $T(\Gamma)$ by finite-dimensional Teichmüller spaces following Gardiner [5].

\section{INVARIANT METRIC GENERATED BY THE GREEN FUNCTION}

Using the negative plurisubharmonic functions, Azukawa [1] introduced an invariant differential (pseudo)metric on complex manifolds $\mathscr{M}$ over $\mathbf{C}^{n}$; his 
construction was extended in [13] to the complex Banach manifolds. Let us denote this metric, determined on the tangent bundle $T \mathscr{M}$ of $\mathscr{M}$, by $A_{\mathscr{M}}(x, \xi)$; here $x \in \mathscr{M}$ and $\xi \in T_{x} \mathscr{M}$ is a tangent vector.

Applying Theorem 1, one can establish the following result:

Theorem 2. For all spaces $T(\Gamma)$,

$$
A_{T(\Gamma)}(x, \xi)=F_{T(\Gamma)}(x, \xi)
$$

where $F_{T(\Gamma)}(x, \xi)$ is the Finsler structure of $T(\Gamma)$. Consequently, the Teichmüller metric is nothing but the integrated form of the Azukawa metric of the space $T(\Gamma)$.

\section{Plurisubharmonic Functions \\ AND EXTREMAL QUASICONFORMAL MAPS}

Theorem 1 allows us to give a new characterization of extremal Beltrami differentials by using plurisubharmonic functions.

Recall that a differential $\mu_{0} \in M(\Gamma)$ is called extremal if $\left\|\mu_{0}\right\|=$ $\inf \left\{\|\mu\|: \Phi_{\Gamma}(\mu)=\Phi_{\Gamma}\left(\mu_{0}\right)\right\}$, i.e., $d_{T(\Gamma)}\left(0, \Phi_{\Gamma}\left(\mu_{0}\right)\right)=d_{M(\Gamma)}\left(0, \mu_{0}\right)$.

Theorem 3. A Beltrami differential $\mu_{0} \in M(\Gamma)$ is extremal if and only if there exists a logarithmically plurisubharmonic function $f: T(\Gamma) \rightarrow[0,1), f(0)=0$, such that the ratio $f(x) /\|x\|$ is bounded in some neighborhood of $x=0$ and one of the following inequalities is satisfied:

$$
\limsup _{|t| \rightarrow 0} \frac{f \circ \Phi_{\Gamma}\left(t \mu_{0} /\left\|\mu_{0}\right\|_{\infty}\right)}{|t|}=1, \quad f \circ \Phi_{\Gamma}\left(\mu_{0}\right)=\left\|\mu_{0}\right\|_{\infty} .
$$

A straightforward consequence of the above theorems is the following wellknown criterion for the extremality which plays an important role in many applications (see, e.g., [5, 6, 8, 15, 18]).

Corollary 5. A differential $\mu_{0} \in M(\Gamma)$ is extremal if and only if

$$
\sup \left\{\frac{1}{2}\left|\iint_{U} \mu_{0}(z) \phi(z) d z \wedge d \bar{z}\right|: \phi \in A_{1}(\Gamma),\|\phi\|=1\right\}=\left\|\mu_{0}\right\|_{\infty} ;
$$

here $A_{1}(\Gamma)=\left\{\phi \in L_{1}(U): \phi\right.$ is holomorphic in $\left.U\right\}$.

In [4] the Royden problem on the holomorphic maps from the disc into the Teichmüller space is solved. Theorem 1 gives another approach to this problem.

Full proofs will appear elsewhere.

\section{ACKNOWLEDGEMENT}

This research began during my stay at the University of Bielefeld, SFB 343 Diskrete Strukturen in der Mathematik, and IHES, Bures-Sur-Ivette. I am grateful for their support.

\section{REFERENCES}

1. K. Azukawa, The invariant pseudo-metric related to negative plurisubharmonic functions, Kodai Math. J. 10 (1987), 83-92.

2. J.-P. Demailly, Mesures de Monge-Ampére et mesures plurisubharmoniques, Math. Z. 194 (1987), 519-564. 
3. C. J. Earle, The Teichmüller distance is differentiable, Duke Math. J. 44 (1977), 389-397.

4. C. J. Earle, I. Kra, and S. L. Krushkal, Holomorphic motions and Teichmüller spaces, Cornell Univ., preprint 1991.

5. F. P. Gardiner, Teichmüller theory and quadratic differentials, Wiley Interscience, New York, 1987.

6. R. S. Hamilton, Extremal quasiconformal mappings with prescribed boundary values, Trans. Amer. Math. Soc. 138 (1969), 399-406.

7. M. Klimek, Extremal plurisubharmonic functions and invariant pseudodistances, Bull. Soc. Math. France 113 (1985), 231-240.

8. S. L. Krushkal, Quasiconformal mappings and Riemann surfaces, Winston, Washington, D.C.; Wiley, New York, 1979.

9. __ Strengthening pseudoconvexity of finite dimensional Teichmüller spaces, Math. Ann. 290 (1991), 681-687.

10. S. L. Krushkal and R. Kühnau, Quasikonforme Abbildungen-neue Methoden und Anwendungen, Teubner-Texte Math., vol. 54, Teubner, Leipzig, 1983.

11. P. Lelong, Fonction de Green pluricomplexe et lemmes de Schwarz dans les espaces de Banach, J. Math. Pures Appl. 69 (1989), 319-347.

12. S. Nag, The complex analytic theory of Teichmüller spaces, Wiley Interscience, New York, 1988.

13. M. Nishihara, K. S. Shon and N. Sugawara, On pseudo-metrics and their indicatrices in balanced open subsets of a locally convex space, Math. Rep. Toyama Univ. 9 (1986), 109-136.

14. E. A. Poletskii and B. V. Shabat, Invariant metrics, Several Complex Variables III, Geometric Function Theory, Encyclopedia Math. Sci. (G.M. Henkin, eds.), 9, Springer, Berlin, Heidelberg and New York, 1989, pp. 63-111.

15. E. Reich and K. Strebel, Extremal quasiconformal mappings with given boundary values, Contribution to Analysis, Academic Press, New York and London, 1974, pp. 375-391.

16. H. L. Royden, Automorphisms and isometries of Teichmüller space, Advances in the Theory of Riemann Surfaces, Ann. of Math. Stud., 66, Princeton Univ. Press, Princeton, NJ, 1971, pp. 369-383.

17. Z. Slodkowski, Holomorphic motions and polynomial hulls, Proc. Amer. Math. Soc. 111 (1991), 374-355.

18. K. Strebel, Extremal quasiconformal mappings, Results in Math. 10 (1986), 169-210.

Research Institute for Mathematical Sciences and Department of Mathematics and COMPUTER SCIENCE, Bar-Illan UNIVERSITY, 52900 Ramat-GaN, ISRAEL

Institute of Mathematics, Siberian Branch of the Russian Academy of Sciences, 630090, NovosibiRsK, RUSSIA 